\title{
PREDICTION OF LIQUID HOLDUP IN COUNTERCURRENT-FLOW ROTATING PACKED BED
}

\author{
CHIA-CHANG LIN, YU-SHAO CHEN and HWAI-SHEN LIU \\ Department of Chemical Engineering, National Taiwan University, Taipei, Taiwan, ROC
}

\begin{abstract}
A theoretical analysis of the liquid holdup in the countercurrent-flow rotating packed bed has been developed. It is based on liquid film on a rotating disk in the presence of the gas, with the assumption that the rotating packed bed may be modelled as $n$ layers of hypothetical rotating disks stacked together. Agreement between the predicted values of the liquid holdup and the experimental values was quite good. With this model, the liquid holdup can be estimated in the countercurrent-flow rotating packed bed with data of pressure drop. Furthermore, a correlation for the liquid holdup in the conventional packed bed was extended and modified to estimate that in the countercurrent-flow rotating packed bed.
\end{abstract}

Keywords: rotating packed bed; liquid holdup; pressure drop; film thickness; wetted surface area

\section{INTRODUCTION}

The utilization of centrifugal force to enhance mass transfer has been well known in many applications. Several centrifugal contractors can be found in the literature. For a gas-liquid system, the rotating packed bed has been discussed during the past few years. This new device was invented by Ramshaw and Mallinson ${ }^{1}$ at ICI and termed 'HIGEE'. During the 1980s, they and co-workers developed a series of the HIGEE process and applied it to absorption and distillation. As the centrifugal acceleration can be much more than the gravitational one, many advantages may be achieved. Due to the reduced tendency of flooding, the system can be operated within a wider range of gas and liquid flow rates. Moreover, the liquid film will become thinner and volumetric mass transfer can also be enhanced. As a result, the size of the equipment would be greatly reduced as compared with the conventional packed bed. Thus, the capital and operating cost will be reduced $\left(\right.$ Ramshaw $^{2}$; Short $\left.{ }^{3}\right)$. These characteristics are very important for chemical processing industries (CPI). Hence, the rotating packed bed plays a particularly important role for process intensification in the industries. In 1986, Glitsch, Inc. (Dallas, TX) obtained from ICI worldwide marketing rights of the HIGEE process (Basta ${ }^{4}$ ). By late 1993 Glitsch introduced HIGEE into the commercial scale for acid gas treatment/dehydration and for stripping volatile organics from ground water (Bucklin and Won ${ }^{5}$; Fowler et al. ${ }^{6}$ ). Some useful fundamental studies of the rotating packed bed can be found in many papers (Keyvani and Gardner'; Singh et al. ${ }^{8}$; Kumar and Rao9; Kelleher and Fair ${ }^{10}$; Basic and Dudukovic ${ }^{11}$; Munjal et al. ${ }^{12}$; Burns and Ramshaw ${ }^{13}$ ).

Although the rotating packed bed can be applied to absorption and distillation, the operating characteristics are understood poorly to date. For future applications, these characteristics must be realized for proper design and operation. A main goal of this work is to develop a hydrodynamics model to predict liquid holdup that is an important characteristic in a rotating packed bed. With pressure drop data or relevent correlations, the liquid holdup may be estimated by the following proposed model. This is very useful for commercial operation because the liquid holdup is more difficult to measure than the pressure drop. More specifically, an important objective of the present work is to extend the correlation of liquid holdup based on the conventional packed bed to a rotating packed bed.

\section{BACKGROUND}

Keyvani and Gardner ${ }^{7}$ thought that the total pressure drop in the rotating packed bed must be clarified into two regions: one is the pressure drop across the bed, the other is the pressure drop between the stationary housing and the spinning rotor. Based on this, they modelled pressure drop by a momentum balance and Ergun's correlation often used in the conventional packed bed. From their results, it can be shown that all of predictions was within $\pm 20 \%$ error for the dry bed. However, for the wet bed, the model overpredicted by $20 \%$. They also found an important phenomena that the wet bed had a lower pressure drop than the dry bed in the same situations. The main parameters that affect the pressure drop included the rotor speed, gas and liquid flows. For the dry bed and the wet bed, the pressure drop increased with the square of the rotor speed at constant gas and liquid flow rates. Certainly, the pressure drop also increased with both gas and liquid flow rates, but the influence of liquid was minor.

They also studied the residence time distribution (RTD) using a stimulus-response technique, and used the mean residence time to estimate the liquid holdup under various conditions. The results showed that, as expected, the mean residence time decreased as the rotor speed increased or the liquid flow rates increased. As for the effect of the gas flow rate, there was a slight effect on the mean residence time within all ranges of gas flow. 
Singh et al. ${ }^{8}$ suggested that the pressure drop across the packing was the sum of rotational pressure drop and frictional pressure drop. With the experimental data, they obtained the following equation.

$$
\Delta P=0.92 \rho_{G} \omega^{2}\left(r_{o}^{2}-r_{i}^{2}\right)+0.99 \rho_{G}\left(\frac{a_{t}}{\varepsilon}\right)\left(r_{o}-r_{i}\right) V_{\text {Gavg }}^{2}
$$

This equation is applicable in the region of higher rotor speed or lower liquid flow rates because of less liquid holdup. As a result, if liquid flow rate is high, equation (1) is not suitable to estimate the pressure drop.

Kumar and $\mathrm{Rao}^{9}$ thought that the pressure drop across the rotor resulted mainly from the centrifugal $\left(\Delta P_{c}\right)$ and frictional force $\left(\Delta P_{f}\right)$ and the kinetic energy $\left(\Delta P_{k}\right)$.

$$
\Delta P=\Delta P_{c}+\Delta P_{f}+\Delta P_{k}
$$

They assumed that liquid flowed in the form of thin film and gas distribution in the bed was uniform. Based on these, some theoretical analysis was obtained.

$$
\begin{aligned}
& \Delta P_{c}=\frac{1}{2} \rho_{G}\left(K_{s} \omega\right)^{2}\left(r_{o}^{2}-r_{i}^{2}\right) \\
& \Delta P_{f}=\frac{1}{2} \rho_{G}\left(K_{a} f\right) r_{o}^{2} \frac{G^{2}}{\rho_{G}} \frac{a_{t}(1-\varepsilon)}{\varepsilon^{3}} \Delta I
\end{aligned}
$$

where

$$
\begin{aligned}
\Delta I & =I\left(r_{o}\right)-I\left(r_{i}\right) \\
I(r) & =3 c^{-3 / 2}\left[\frac{1}{16} \ln \left(\frac{r^{1 / 3}+c^{1 / 2}}{r^{1 / 3}-c^{1 / 2}}\right)-\frac{1}{8} c^{1 / 2} r^{1 / 3} \frac{r^{2 / 3}+c}{\left(r^{2 / 3}-c\right)^{2}}\right] \\
c & =\frac{n}{\varepsilon Z}\left[\frac{3 \mu_{L} L}{2 \pi \rho_{L}^{2} \omega^{2} n}\right]^{1 / 3}
\end{aligned}
$$

$K_{s}$ and $K_{a} f$ must be estimated from the experimental data. The pressure drop was measured across the gas inlet and outlet; therefore, $\Delta P_{k}$ was not considered. They obtained values of $K_{s}$ of 0.73 and 0.51 for rotor speeds of 610 and $1150 \mathrm{rpm}$, respectively. With the rotational Reynolds number, $R e_{\theta}, K_{a} f$ can be estimated by the following equation.

$$
\log \left(\frac{1}{K_{a} f}\right)=1.78+1.42 \times 10^{-5} R e_{\theta}+1.6 \log R e_{G}
$$

The deviations between the estimated and experimental $\Delta P$ values were within $\pm 20 \%$.

Kelleher and Fair ${ }^{10}$ also studied the pressure drop in the rotating packed bed, and presented the following model.

$$
\Delta P=\frac{\rho_{G} \omega^{2}}{2}\left(r_{o}^{2}-r_{i}^{2}\right)+\frac{5 B^{\prime}}{22}\left(\frac{\varepsilon M_{G}}{\pi Z_{b} \rho_{G}}\right)^{2}\left(\frac{1}{r_{i}^{1 / 10}}-\frac{1}{r_{o}^{11 / 10}}\right)
$$

This equation implied that the pressure drop across the rotating packed bed was made up of two components: a centrifugal force term and a high Reynolds number term. Although equation (8) did not include any empirical parameters, it is not applicable in high liquid-to-gas ratios or the region where the liquid effect appears to be major.

Basic and Dudukovic ${ }^{11}$ presented the very first direct experimental data for the liquid holdup in the rotating packed bed, using the conductance method. They established an experimental setup as well as a relationship between bed conductance and liquid holdup. Thus, with the electrical signal from the rotating packed bed, the liquid holdup in the bed may be estimated. They found liquid holdup dependent on the liquid flow rate and rotor speed; the increase of the latter led to less liquid holdup. They also showed that holdup cannot be interpreted by the film flow model based on the assumptions that liquid is the only flowing phase, and the packing surface is completely wetted. However, these assumptions are unlikely to be valid in real flow phenomena. Thus, the film flow model derived by them failed to predict liquid holdup consequently.

In conventional packed beds, the relationship between the liquid holdup and the pressure drop was investigated widely in the open literature. However, for the rotating packed bed, very little information has been proposed, as indicated. Consequently, this work is to develop a model to relate the liquid holdup and the pressure drop in a rotating packed bed with both gas and liquid flows. As a result, the pressure drop correlations can be used as described above to predict liquid holdup in the rotating packed bed.

\section{MODEL DEVELOPMENT}

Munjal et al. ${ }^{12}$ presented a model based on the momentum integral method to predict the film thickness and the velocity profiles in the rotating packed bed. Although it may be applicable for mass-transfer in the rotating packed bed, it only can predict liquid holdup for the case of no gas flow or low gas flow rate. It must be modified by introducing the gas flow effect. The rotating packed bed may be visualized as a stack of rotating disks (as illustrated in Figure 1); hence, the hydrodynamics could be considered over a rotating disk. The velocity profile and thickness of the liquid film on a rotating disk have been studied by several investigators (such as Matsumoto et al. ${ }^{14}$; Thomas et al. ${ }^{15}$ ). However, they only analysed liquid film in the absence of the gas.

The condition that gas flows over the free liquid surface on the rotating disk may be considered (as shown in Figure 2 ). The liquid velocity in the film is assumed to be independent of $\theta$. The equations of continuity and motion for a constant-density liquid may be written as

$$
\begin{aligned}
& \frac{\partial V_{r}}{\partial r}+\frac{V_{r}}{r}+\frac{\partial V_{z}}{\partial z}=0 \\
& V_{r} \frac{\partial V_{r}}{\partial r}-\frac{V_{\theta}^{2}}{r}+V_{z} \frac{\partial V_{r}}{\partial z}=v_{L} \frac{\partial^{2} V_{r}}{\partial z^{2}}-\frac{1}{\rho_{L}} \frac{\partial P}{\partial r} \\
& V_{r} \frac{\partial V_{\theta}}{\partial r}+\frac{V_{r} V_{\theta}}{r}+V_{z} \frac{\partial V_{\theta}}{\partial z}=v_{L} \frac{\partial^{2} V_{\theta}}{\partial z^{2}}
\end{aligned}
$$

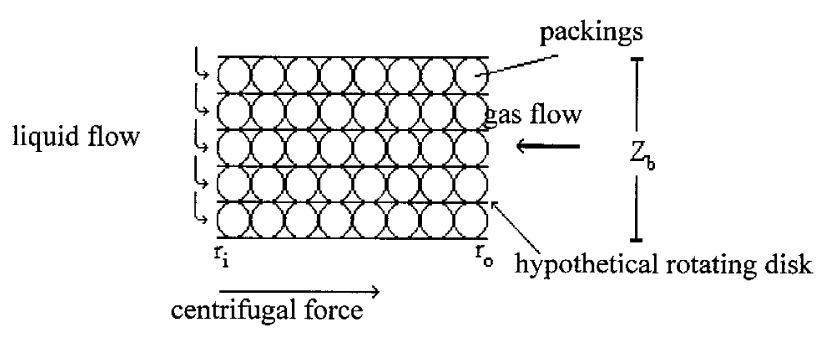

Figure 1. Hypothetical image of the rotating packed bed. 


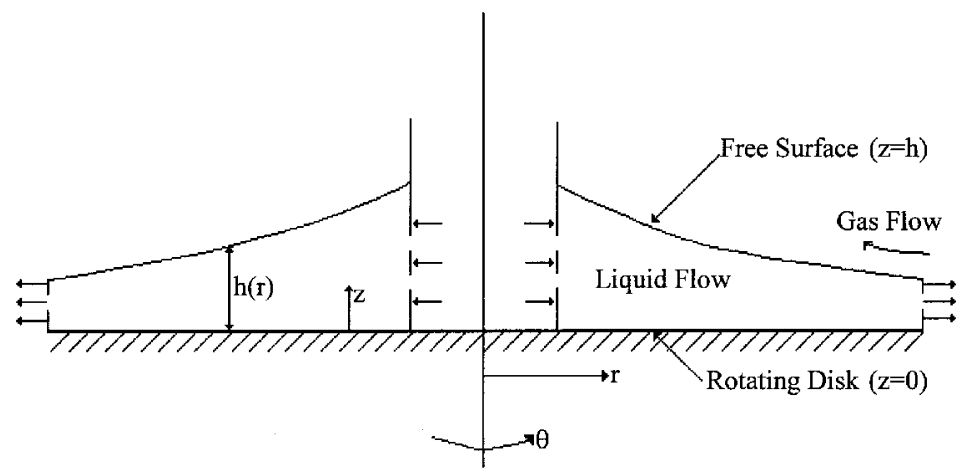

Figure 2. Schematic of flow over the rotating disk.

where $V_{r}$ is the velocity in the radial direction, $V_{z}$ is the velocity in the axial direction which is perpendicular to the disk surface, $V_{\theta}$ is the velocity in the tangential direction, and $\partial P / \partial r$ is caused by the gas. In equations (10) and (11), the terms $v_{L}(\partial / \partial r)\left\{(1 / r)(\partial / \partial r)\left(r V_{r}\right)\right\}$ and $v_{L}(\partial / \partial r)\{(1 / r)(\partial / \partial r)$ $\left.\left(r V_{\theta}\right)\right\}$ have been omitted because these terms are an order of magnitude small than the terms on the right-hand side. The boundary conditions are given by at the disk surface $(z=0)$

$$
\begin{aligned}
& V_{r}=0 \\
& V_{\theta}=r \omega \\
& V_{z}=0
\end{aligned}
$$

at the free surface $(z=h)$

$$
\begin{aligned}
& \frac{\partial V_{r}}{\partial z}=0 \\
& \frac{\partial V_{\theta}}{\partial z}=0
\end{aligned}
$$

To overcome the difficulty of numerical computation, this problem should be simplified by assuming a velocity profile similar to that of the boundary layer over the flat plate:

$$
\begin{aligned}
& V_{r}=V_{r}^{\infty}\left(\frac{3}{2} \frac{z}{h}-\frac{1}{2} \frac{z^{3}}{h^{3}}\right) \\
& V_{\theta}=V_{\theta}^{\infty}\left(\frac{3}{2} \frac{z}{h}-\frac{1}{2} \frac{z^{3}}{h^{3}}\right)+r \omega
\end{aligned}
$$

where $V_{r}^{\infty}$ is the radial velocity at the free surface (i.e. gasliquid interface) and only depends on $r, V_{\theta}^{\infty}$ is the tangential velocity at the free surface, relative to the disk surface, and also only depends on $r$. The above equations satisfy four out of the five boundary conditions [equations (12a), (12b), (13a) and (13b)]. Also, the axial velocity can be obtained by integating equation (9) and using equation (12c)

$$
V_{z}=-\int_{0}^{z}\left(\frac{\partial V_{r}}{\partial r}+\frac{V_{r}}{r}\right) \mathrm{d} z
$$

It is assumed that the rotating packed bed consists of $n$ layers of hypothetical rotating disks, therefore, the volumetric liquid flow rate, $Q$, can be written as

$$
Q=n\left(2 \pi r \int_{0}^{h} V_{r} \mathrm{~d} z\right)
$$

where $n$ is the number of the hypothetical rotating disks and is approximately equal to the ratio of the height of the bed and the diameter of the packing, $Z_{b} / d_{p}$. From the above equation and equation (14a), the estimated thickness of the liquid film in the bed can be obtained:

$$
h=\frac{4 Q d_{p}}{5 \pi r V_{r}^{\infty} Z_{b}}
$$

Substituting equations (14a), (14b) and (15) into equations (10) and (11), with equation (17) and integrating the resulting equations with respect to $z$ between $z=0$ and $z=h$, the following two equations may be obtained for the velocity at the free liquid surface:

$$
\begin{aligned}
\frac{\mathrm{d} V_{r}^{\infty}}{\mathrm{d} r}= & \frac{V_{\theta}^{\infty}}{V_{r}^{\infty} r}+\frac{175}{68} \frac{V_{\theta}^{\infty} \omega}{V_{r}^{\infty}}+\frac{35}{17} \frac{r \omega^{2}}{V_{r}^{\infty}}-\frac{105}{34} \frac{v_{L}}{h^{2}} \\
& +\frac{35}{17} \frac{1}{V_{r}^{\infty} \rho_{L}}\left(-\frac{\mathrm{d} P}{\mathrm{~d} r}\right) \\
\frac{\mathrm{d} V_{\theta}^{\infty}}{\mathrm{d} r}= & -\frac{175}{68} \omega-\frac{V_{\theta}^{\infty}}{r}-\frac{105}{34} \frac{v_{L} V_{\theta}^{\infty}}{V_{r}^{\infty} h^{2}}
\end{aligned}
$$

where $\mathrm{d} P / \mathrm{d} r$ may be evaluated by the following equation based on the research of Keyvani and Gardner ${ }^{7}$ without any experimental data of pressure drop.

$$
\frac{\mathrm{d} P}{\mathrm{~d} r}=\rho_{G} \omega^{2} r-\rho_{G} \frac{\bar{V}_{G}}{\varepsilon^{2}} \frac{\mathrm{d} \bar{V}_{G}}{\mathrm{~d} r}+A \bar{V}_{G}+B \bar{V}_{G}^{2}
$$

where $\bar{V}_{G}$ is the superficial gas velocity, defined as $M_{G} / 2 \pi r Z_{b} \rho_{G} ; A$ and $B$ are the constants of Morton's $\operatorname{model}^{16}$.

$$
\begin{aligned}
& A=\frac{8.5 \mu_{G} a_{t}^{2}}{\varepsilon^{3}} \\
& B=\frac{a_{t} \rho_{G}}{\varepsilon^{3}} \operatorname{Re}^{-0.1}=\frac{a_{t} \rho_{G}}{\varepsilon^{3}}\left(\frac{M_{G}}{2 \pi r Z_{b} a_{t} \mu_{G}}\right)^{-0.1}=B^{\prime} r^{0.1}
\end{aligned}
$$

With a further computation, equation (20) can be rewritten as the following equation.

$$
\frac{\mathrm{d} P}{\mathrm{~d} r}=\rho_{G} \omega^{2} r+\rho_{G}\left(\frac{M_{G}}{2 \pi Z_{b} \rho_{G} \varepsilon}\right)^{2} \frac{1}{r^{3}}+A \bar{V}_{G}+B^{\prime} r^{0.1} \bar{V}_{G}^{2}
$$

If the correlations of the pressure drop are as in equations (1) and (8), $\mathrm{d} P / \mathrm{d} r$ may be considered as $\Delta P /\left(r_{o}-r_{i}\right)$. As a result, for any given initial condition $\left(V_{r}^{\infty}=V_{r, 0}^{\infty}\right.$ and $V_{\theta}^{\infty}=V_{\theta, 0}^{\infty}$ at $r=r_{i}$ ), equations (18) and (19) can be solved 
to obtain the values of $V_{r}^{\infty}$ and $V_{\theta}^{\infty}$ numerically. Then with equations (14a) and (14b), the velocity profile in the bed can be calculated. For known values of $V_{r}^{\infty}$, equation (17) can be used to calculate the estimated thickness of the liquid film as a function of $r$.

Matsumoto et al. ${ }^{14}$ used Navier-Stokes equations in cylindrical coordinates by introducing the dimensionless parameters to obtain the relationships between the radial, the tangential and the axial velocity distributions. These relationships were similar to equations (9), (10) and (11), but were written in the dimensionless form without the effect of pressure drop. They adopted the polynomial approximation of higher degrees for the components of velocity profiles. The resultant solutions for the velocity components give a fair prediction of the thickness of liquid film in comparison with the experimental data. Their solutions may be applied to the rotating packed bed by introducing equation (16) to obtain the following relationship.

$$
\frac{Q d_{p}}{2 \pi r^{2} \sqrt{v \omega Z_{b}}}=\frac{1}{2} a_{1} h_{o}^{2}-\frac{1}{6} h_{o}^{3}-\frac{1}{12} b_{1} h_{o}^{4}-\frac{1}{60} b_{1} h_{o}^{5}
$$

where $h_{o}$ is defined as $h \sqrt{\omega / v}$ and the algebraic equations with respect $a_{1}$ and $b_{1}$ are given by

$$
\begin{aligned}
& a_{1}-h_{o}-b_{1} h_{o}^{2}-\frac{1}{3} b_{1} h_{o}^{3}=0 \\
& b_{1}+a_{1} h_{o}^{2}+\frac{1}{3}\left(a_{1} b_{1}-1\right)=0
\end{aligned}
$$

From equations (22), (23) and (24), the estimated thickness of the liquid film as a function of $r$ can be predicted for the case of the liquid phase.

\section{RESULTS AND DISCUSSION}

The liquid holdup data of Basic ${ }^{17}$ in a rotating packed bed may be used to verify the model. The investigated rotating packed bed had an axial height of $2.54 \mathrm{~cm}$ and the inner, outer radii were 3.14 and $8.54 \mathrm{~cm}$, respectively. The packing was glass beads of $d_{p}=3 \mathrm{~mm}$ operated with an air-water fluid combination. Figure 3 shows a comparison of the estimated film thickness calculated by equations (17) and (22) ignoring the gas (i.e. $\mathrm{d} P / \mathrm{d} r=0$ ). It is well known that the three curves start with a different estimated film thickness but quickly merge to a single curve. Although the estimated film thickness calculated by equation (17) depends on the initial radial velocity, as shown in Figure 3, it is seen clearly that the estimated film thickness is not very sensitive to the initial condition except for a very short 'transition region'. The estimated film thickness increases from the entrance location, attains a peak and then monotonically decreases along the radial direction. The increase in the estimated film thickness near the entrance is due to the strong frictional resistance which resists the inertial and centrifugal forces in that region, and decreases the radial velocity. The centrifugal force, however, increases with radial distance and overcomes the frictional resistance. As a result, the estimated film thickness decreases monotonically. This trend was also observed by Thomas et al. ${ }^{15}$ who presented experimental data for the film thickness distribution at various flowrates and rotational

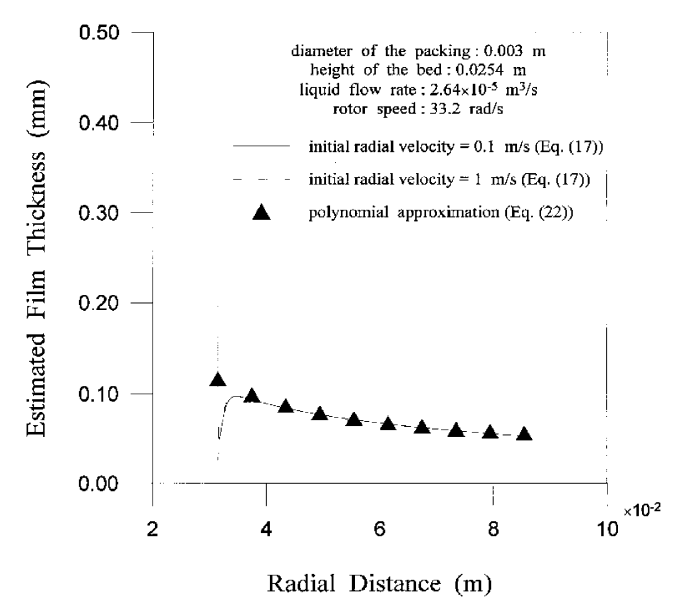

Figure 3. Comparison of the estimated film thickness predicted by two methods in the rotating packed bed in the absence of the gas $\left(V_{\theta, 0}^{\infty}=5 \mathrm{~m} \mathrm{~s}^{-1}\right)$.

speeds. However, the polynomial approximation predicts a monotonical decrease in the estimated film thickness along the radial direction. Also, at the distant radial location, the estimated film thickness predicted by both models approaches a similar value because the flow becomes mainly driven by the centrifugal force.

The variations of the estimated film thickness along the radial direction at the lower liquid flow rate without gas are shown in Figure 4 for various rotor speeds. It is shown that the estimated film thickness increases near the entrance, attains a peak and then decreases further away from the inlet. The location of the peak decreases with the increase in the rotor speed. This can be explained by the fact that the lower rotor speed requires the longer distance to resist the frictional force from the surface. However, with higher liquid flow rate, the effect of the inertial force is more dominant than that of the centrifugal force. As a result, at lower rotor speeds, the estimated film thickness decreases near the entrance and then increases later along the radial distance, as shown in Figure 5. Also, when the centrifugal force is dominant (i.e. the higher rotor speed), the estimated film thickness decreases further downstream. Based on the

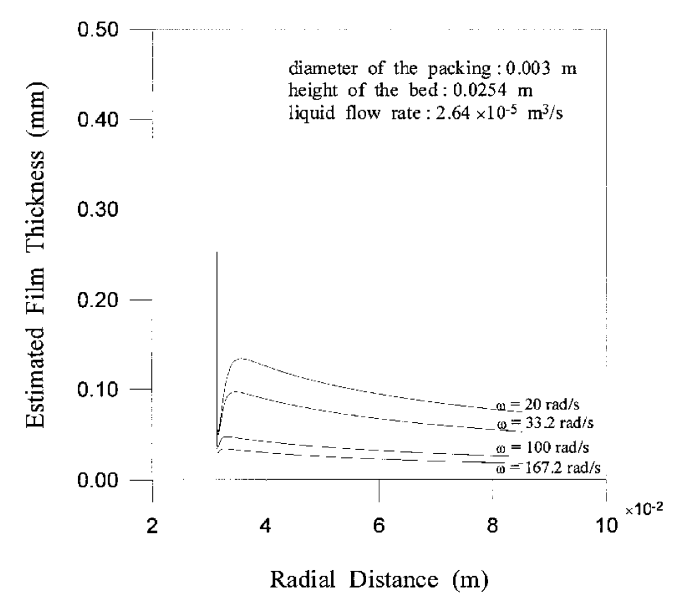

Figure 4. Comparison of the estimated film thickness distributions for various rotor speeds at lower liquid flow rate in the absence of the gas $\left(V_{r, 0}^{\infty}=0.1 \mathrm{~m} \mathrm{~s}^{-1}, V_{\theta, 0}^{\infty}=5 \mathrm{~m} \mathrm{~s}^{-1}\right)$. 


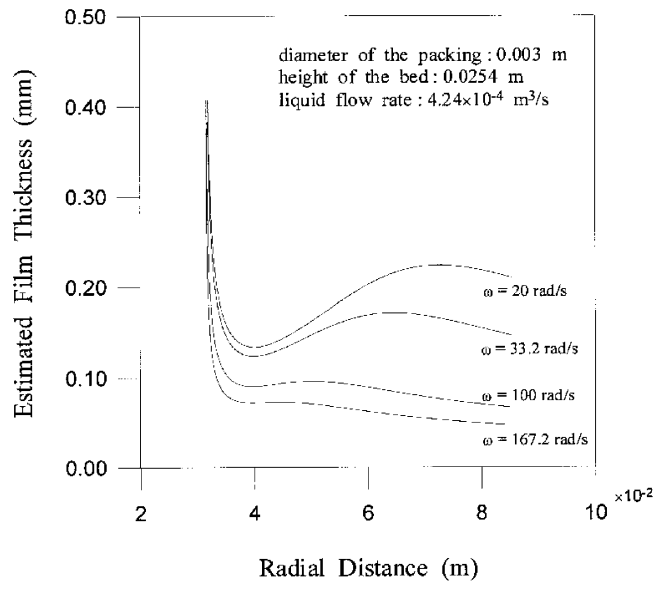

Figure 5. Comparison of the estimated film thickness distributions for various rotor speeds at higher liquid flow rate in the absence of the gas $\left(V_{r, 0}^{\infty}=0.1 \mathrm{~m} \mathrm{~s}^{-1}, V_{\theta, 0}^{\infty}=5 \mathrm{~m} \mathrm{~s}^{-1}\right)$.

above analysis, it is well realized that the forces affecting the estimated film thickness are the inertial force, the frictional force, and the centrifugal force. Also, it is found that the pattern of the film for various conditions is quite complex due to the relative magnitude of three forces that may affect the hydrodynamics over the rotating disk.

The polynomial approximation method cannot predict the estimated film thickness affected by the gas, as shown in Figure 6. At a short radial distance, the polynomial approximation method underpredicts the estimated film thickness due to the effect of gas. Because the frictional resistance from the surface has to overcome the inertial and centrifugal force, and also the gas flow, the peak is higher than the case without gas. While further away from the entrance, the flow is mainly determined by the centrifugal force, so the estimated film thickness by the polynomial approximation approaches that of the above model (equation (17)) as that in the absence of the gas. Therefore, the above model (equation (17)) should have better applicability even though the polynomial approximation can also

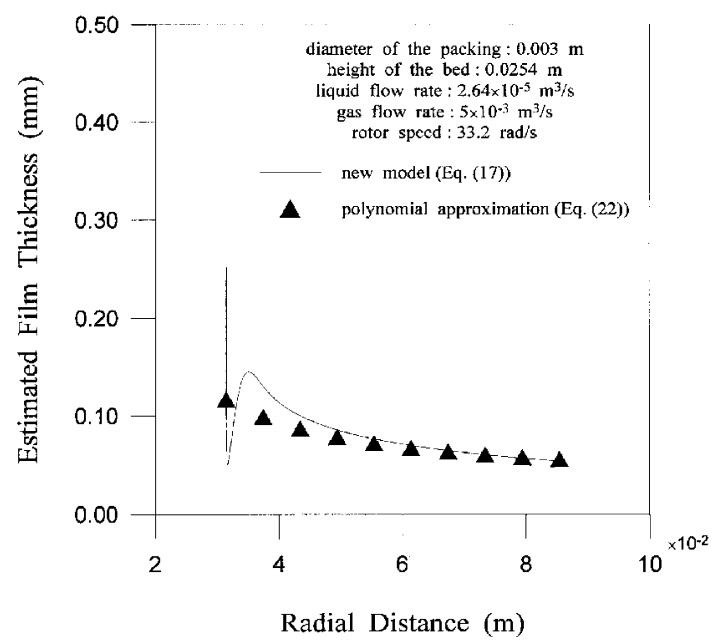

Figure 6. Comparison of the estimated film thickness predicted by two methods in the rotating packed bed in the presence of the gas $\left(V_{r, 0}^{\infty}=0.1 \mathrm{~m} \mathrm{~s}^{-1}, V_{\theta, 0}^{\infty}=5 \mathrm{~m} \mathrm{~s}^{-1}\right)$. provide a good prediction for the estimated film thickness in the absence of the gas.

The mean estimated thickness of the liquid film, $\bar{h}$, can be defined as

$$
\bar{h}=\frac{1}{r_{o}-r_{i}} \int_{r_{i}}^{r_{o}} h \mathrm{~d} r
$$

The real flow behaviour can be visualized by including the fact that the surface area of the packing is not completely wetted for various conditions encountered in practice. As a result, the following relationship exists between the liquid holdup, $h_{L}$, and the wetted surface area, $a_{w}$ :

$$
h_{L}=\bar{h} \times a_{w}
$$

To predict liquid holdup, the dependence of $\bar{h}$ and $a_{w}$ on the various operating conditions must be known. Based on the above description, $\bar{h}$ can be found for any conditions from equations (17) and (25). However, in the rotating packed bed, there is no information for the wetted surface area in the literature. Consequently, a correlation must be developed for the wetted surface area while liquid is the only flowing phase (i.e. $\mathrm{d} P / \mathrm{d} r=0$ ). With the experimental data for liquid holdup from Basic $^{17}$ and the mean liquid estimated film thickness obtained by equations (17) and (25), the values of the wetted surface area can be obtained for various conditions by using equation (26), then the following correlation is derived.

$$
\frac{a_{w}}{a_{t}}=584 R e_{L}^{-1.03} W e_{L}^{0.576} \mathrm{Fr}_{L}^{0.123}
$$

Figure 7 shows that the predicted liquid holdup using equations (17), (26) and (27) is very close to the experimental liquid holdup when the liquid is the only flowing phase. When the general case of the two-phase flow of both gas and liquid is considered, the liquid holdup will be in a different form from that in the one-phase flow. As a result, equations (17) and (25) are used to predict the mean estimated liquid film thickness, considering the effect of the pressure drop (i.e. the effect of the gas), and then equation (27) is used to obtain the liquid holdup for the two-phase flow by assuming a similar wetted area for both one-phase and two-phase flows. As shown in Figure 8, the results

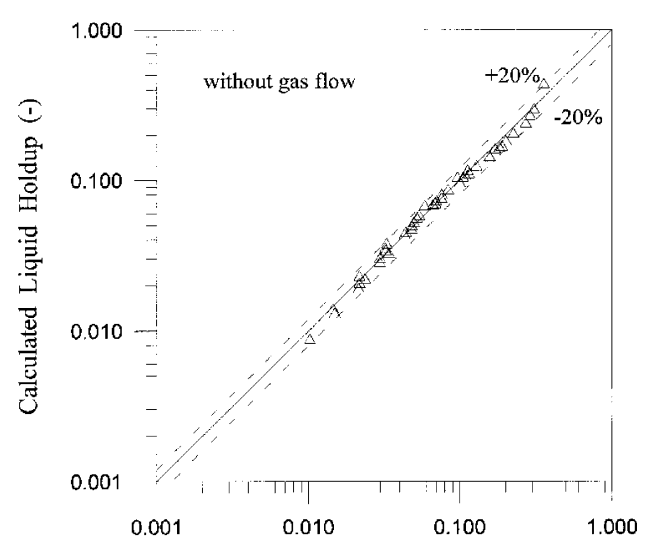

Experimental Liquid Holdup (-)

Figure 7. Comparison of experimental and predicted liquid holdup in the absence of gas (model: equations (17), (26) and (27); data from Basic ${ }^{17}$ ). 


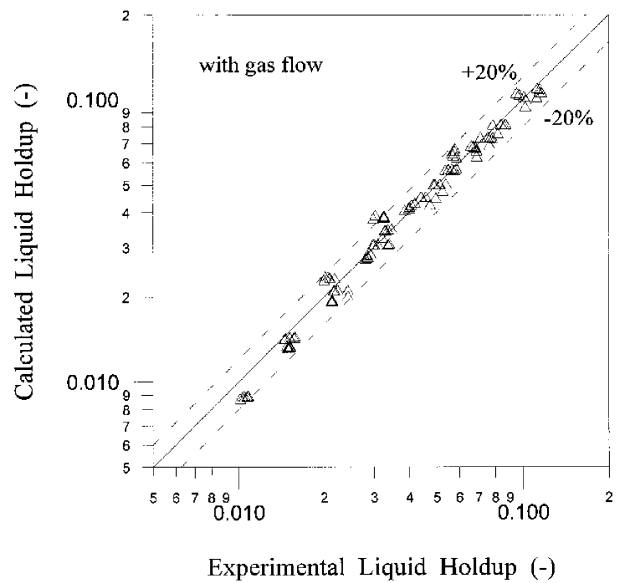

Figure 8. Comparison of experimental and predicted liquid holdup in the presence of gas (model: equations (17), (26) and (27); data from Basic ${ }^{17}$ ).

predicted by equations (17), (26) and (27) lie within $\pm 20 \%$ of the experimental data. Thus, this new model is acceptable to predict the liquid holdup with the experimental data of the pressure drop.

\section{APPLICATIONS}

If the pressure drop in a rotating packed bed is known, the liquid holdup can be calculated using the above method (i.e. equations (17), (26) and (27)). Liu et al. ${ }^{18}$ has investigated a rotating packed bed of axial height $2.5 \mathrm{~cm}$ and inner, outer radius of 4.5 and $7 \mathrm{~cm}$, respectively. They presented some results of the pressure drop for an air-water system. With those data, the liquid holdup can be estimated for various operating conditions. As shown in Figure 9, it is realized that the liquid holdup is affected by the gas velocity and increases with the gas rate. This result agrees with the findings of some authors who studied the hydrodynamics in the conventional packed bed. It is also seen that the liquid holdup and the pressure drop have a similar tendency.

For the conventional packed bed of the low interaction regime where the flow of one phase is littlely affected by the other phase, Specchia and Baldi ${ }^{19}$ thought that the hydrodynamics of the liquid on the packing is very different

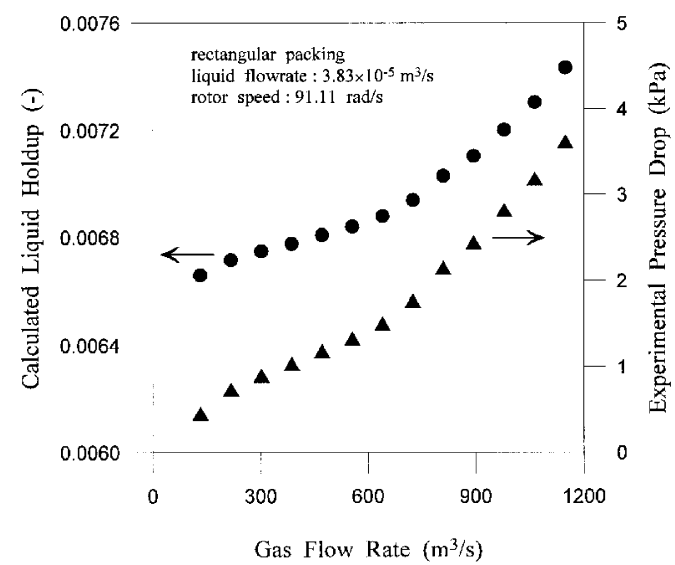

Figure 9. Effect of gas flow rate on liquid holdup (liquid holdup calculated by equations (17), (26) and (27); pressure drop data from Liu et al. ${ }^{18}$ ).

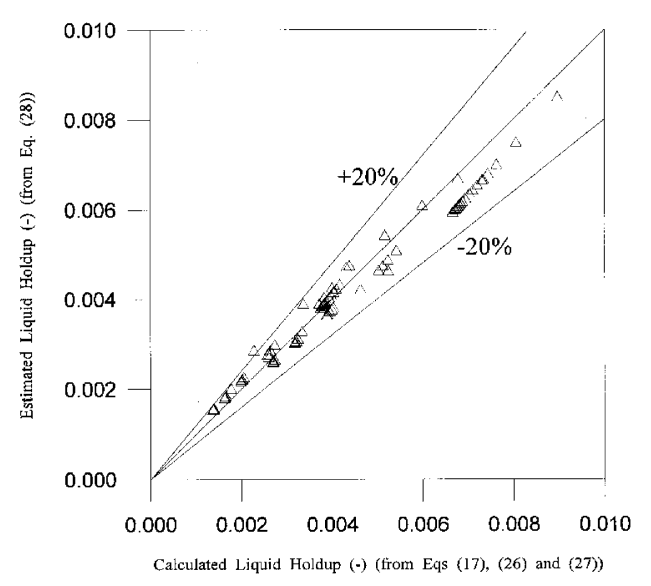

Figure 10. Comparison of liquid holdup estimated by equation (28) and equations (17), (26) and (27) (pressure drop data from Liu et al. ${ }^{18}$ ).

from that of the free liquid. Hence, a general correlation for the liquid holdup in co-current two-phase flow can be obtained by introducing the modified Galileo number $\left(G a^{*}\right)$ :

$$
h_{L}=3.86 R e_{L d}^{0.545} G a^{*-0.42}\left(\frac{a_{t} d_{p}}{\varepsilon}\right)^{0.65} \varepsilon
$$

where $G a^{*}$ is defined by:

$$
G a^{*}=\frac{d_{p}^{3} \rho_{L}\left(\rho_{L} g+\frac{\Delta P}{Z}\right)}{\mu_{L}^{2}}
$$

$G a^{*}$ may be modified in equation (28) to predict the liquid holdup in the rotating packed bed as the following equation, since the flow direction of gas and liquid is inverse.

$$
G a^{*}=\frac{d_{p}^{3} \rho_{L}\left(\rho_{L} a_{c}-\frac{\Delta P}{r_{o}-r_{i}}\right)}{\mu_{L}^{2}}
$$

It is noted clearly that this correlation takes into account the influence of the pressure drop on the liquid holdup. This can be demonstrated by the comparison of this correlation (equation (28)) with the predictions by the model (equations (17), (26) and (27)) for two-phase flow. This comparison is shown in Figure 10 for two-phase flow with the pressure drop data from Liu et al. ${ }^{18}$. When the constant (3.86) in equation (28) is replaced with 1.2 , the discrepancy between this correlation and the prediction is within $\pm 20 \%$, as shown in Figure 10. This implies that the liquid holdup in the rotating packed bed is less than that in the conventional packed. Consequently, this often used correlation seems to be able to predict the liquid holdup in the rotating packed bed with two-phase flow.

\section{CONCLUSIONS}

Because the liquid holdup is very difficult to measure in the rotating packed bed, it would be useful to find another method to obtain the liquid holdup. Based on the analysis of the above, a model has been developed to predict the liquid holdup using the pressure drop data which is easier to measure. It is also evident that the liquid holdup is influenced slightly by the gas. Moreover, the correlation 
(equation (28)) for the liquid holdup in the conventional packed bed can be extended to estimate the liquid holdup in the rotating packed bed.

\section{NOMENCLATURE}

$A, B, \quad$ constants in equation (20)

$B^{\prime} \quad$ constants in equation (8)

$a_{1}, b_{1}$ defined by equations (23) and (24)

$a_{c} \quad$ centrifugal acceleration, $\mathrm{m} \mathrm{s}^{-2},=\omega^{2}\left(r_{i}+r_{o}\right) / 2$

$a_{t} \quad$ total specific surface area of the packing, $\mathrm{m}^{2} \mathrm{~m}^{-3}$

$a_{w} \quad$ wetted surface area of the packing, $\mathrm{m}^{2} \mathrm{~m}^{-3}$

$c \quad$ defined by equation (6)

$d_{p} \quad$ spherical equivalent diameter of the packing, $\mathrm{m},=6(1-\varepsilon) / a_{t}$

$G \quad$ gas flow rate, $\mathrm{kg} \mathrm{m}^{-2} \mathrm{~s}^{-1}$

gravitational acceleration, $\mathrm{m} \mathrm{s}^{-2}$

estimated liquid film thickness, $\mathrm{m}$

mean estimated liquid film thickness, $\mathrm{m}$

liquid holdup (-)

$I(r) \quad$ defined by equation (5)

$K_{s} \quad$ slip factor (-)

$K_{a} f \quad$ defined by equation (7)

$M_{G} \quad$ mass flow rate of gas, $\mathrm{kg} \mathrm{s}^{-1}$

$L \quad$ liquid flow rate, $\mathrm{kg} \mathrm{s}^{-1}$

$n \quad$ number of rotating disks

$P \quad$ pressure of gas, $\mathrm{Pa}$

$\Delta P \quad$ pressure drop, $\mathrm{Pa}$

$\Delta P_{c} \quad$ pressure drop due to centrifugal force, $\mathrm{Pa}$

$\Delta P \quad$ pressure drop due to frictional force, $\mathrm{Pa}$

$\Delta P_{k}{ }_{k} \quad$ pressure difference due to change in gas velocity, $\mathrm{Pa}$

$Q \quad$ volumetric flow rate of liquid, $\mathrm{m}^{3} \mathrm{~s}^{-1}$

$R_{h} \quad$ hydraulic radius, $\mathrm{m}$

$r \quad$ coordinate direction parallel to disk surface

$r_{i} \quad$ inner radius of the packed bed, $\mathrm{m}$

$r_{o} \quad$ outer radius of the packed bed, $\mathrm{m}$

$u_{L} \quad$ superficial liquid velocity, $\mathrm{m} \mathrm{s}^{-1}$

$V_{\text {Gavg }} \quad$ average superficial gas velocity, $\mathrm{m} \mathrm{s}^{-1}$

$\bar{V}_{G} \quad$ superficial gas velocity, $\mathrm{m} \mathrm{s}^{-1}$

$V_{r} \quad$ liquid velocity in the radial direction, $\mathrm{m} \mathrm{s}^{-1}$

$V_{r}^{\infty} \quad$ radial velocity at the gas-liquid surface, $\mathrm{m} \mathrm{s}^{-1}$

$V_{r, 0}^{\infty} \quad V_{r}^{\infty}$ at $r=r_{i} \mathrm{~m} \mathrm{~s}^{-1}$

$V_{z}^{r, 0} \quad$ liquid velocity in the axial direction, $\mathrm{m} \mathrm{s}^{-1}$

$V_{\theta} \quad$ liquid velocity in the tangential direction, $\mathrm{m} \mathrm{s}^{-1}$

$V_{\theta}^{\infty} \quad$ tangential velocity at the gas-liquid surface relative to the disk surface, $\mathrm{m} \mathrm{s}^{-1}$

$V_{\theta, 0}^{\infty} \quad V_{\theta}^{\infty}$ at $r=r_{i}, \mathrm{~m} \mathrm{~s}^{-1}$

$z \quad$ coordinate direction perpendicular to disk surface

$Z_{b} \quad$ axial height of the packing, $m$

$Z \quad$ height of the conventional packed bed, $m$

\section{Greek letters}

voidage (-)

gas viscosity, $\mathrm{kg} \mathrm{m}^{-1} \mathrm{~s}^{-1}$

liquid viscosity, $\mathrm{kg} \mathrm{m}^{-1} \mathrm{~s}^{-1}$

gas density, $\mathrm{kg} \mathrm{m}^{-3}$

liquid density, $\mathrm{kg} \mathrm{m}^{-3}$

dynamic gas viscosity, $\mathrm{m}^{2} \mathrm{~s}^{-1}$

dynamic liquid viscosity, $\mathrm{m}^{2} \mathrm{~s}^{-1}$

surface tension of liquid, $\mathrm{Nm}^{-1}$

angular velocity, $\mathrm{rad} \mathrm{s}^{-1}$

\section{Dimensionless groups}

$\mathrm{Fr}_{L} \quad$ Froude number $\left(u_{L}^{2} a_{t} / a_{c}\right)$

$\mathrm{Gr}^{*} \quad$ Grashof number for liquid defined by equation (29) or equation (30)

$R e_{G} \quad$ gas Reynolds number $\left(4 R_{h} G / \mu_{G}\right)$

$R e_{L} \quad$ liquid Reynolds number $\left(u_{L} / a_{t} v_{L}\right)$

$R e_{L d} \quad$ liquid Reynolds number $\left(u_{L} d_{p} / v_{L}\right)$
$R e_{\theta} \quad$ rotational Reynolds number $\left(K_{s} \omega r_{o}^{2} / v_{G}\right)$

$W e_{L} \quad$ Weber number $\left(u_{L}^{2} \rho_{L} / a_{t} \sigma\right)$

\section{REFERENCES}

1. Ramshaw, C. and Mallinson, R. H., 1981, Mass transfer process, US Patent 4,283,255.

2. Ramshaw, C., 1983, HIGEE distillation-An example of process intensification, Chem Eng, 13-14 Feb.

3. Short, H., 1983, New mass-transfer find is a matter of gravity, Chem Eng, 23-29 Feb.

4. Basta, N., 1987, Facelift for distillation, Chem Eng, 14-16 Mar.

5. Bucklin, R. W. and Won, K. W., 1987, Higee contactors for selective $\mathrm{H}_{2} \mathrm{~S}$ removal and superdehydration, Laurance Reid Gas Conditioning Conf, University of Oklahoma, March 2-4.

6. Fowler, R., Gendes, K. F. and Nyguard, H. F., 1989, Commercial scale demonstration of Higee for $\mathrm{CO}_{2} / \mathrm{H}_{2} \mathrm{O}$ removal, 21st Annual Offshore Technology Conf, Houston, TX, May.

7. Keyvani, M. and Gardner, N. C., 1989, Operating characteristics of rotating beds, Chem Eng Prog, 85: 48-52.

8. Singh, S. P., Wilson, J. H., Counce, R. M., Villiers-Fisher, J. F., Jennings, H. L., Lucero, A. J., Reed, G. D., Ashworth, R. A. and Elliott, M. G., 1992, Removal of volatile organic compounds from groundwater using a rotary air stripper, Ind Eng Chem Res, 31: 574-580.

9. Kumar, M. P. and Rao, D. P., 1990, Studies on a high-gravity gas-liquid contactor, Ind Eng Chem Res, 29: 917-920.

10. Kelleher, T. and Fair, J. R., 1996, Distillation studies in a high-gravity contactor, Ind Eng Chem Res, 35: 4646-4655.

11. Basic, A. and Dudukovic, M. P., 1995, Liquid holdup in rotating packed beds: Examination of the film flow assumption, AIChE J, 41: 301-316.

12. Munjal, S., Dudukovic, M. P. and Ramachandran, P. A., 1989, Mass transfer in rotating packed beds: I. Development of gas-liquid and liquid-solid mass-transfer coefficients, Chem Eng Sci, 44: 2245-2256.

13. Burns, J. R. and Ramshaw, C., 1996, Process intensification: Visual study of liquid maldistribution in rotating packed beds, Chem Eng Sci, 51: $1347-1352$.

14. Matsumoto, S., Saito, K. and Takashima, Y., 1973, The thickness of a viscous liquid film on a rotating risk, J Chem Eng Japan, 6: 503-507.

15. Thomas, S., Faghri, A. and Hankey, W., 1991, Experimental analysis and flow visualization of thin liquid film on a stationary and rotating disk, ASME J Fluids Eng, 113: 73-80.

16. Morton, F., King, P. J. and Atkinson, B., 1964, Operating characteristics of packed columns Part I: Below the "load" point, Trans IChemE, 42: T35-T43.

17. Basic, A., 1992, Liquid holdup and hydrodynamics of rotating packed beds, PhD Diss (Department of Chemical Engineering, Washington University, St. Louis, MO).

18. Liu, H. S., Lin, C. H., Wu, S. C. and Hsu H. W., 1996, Characteristics of a rotating packed bed, Ind Eng Chem Res, 35: 3590-3596.

19. Specchia, V. and Baldi, G., 1977, Pressure drop and liquid holdup for two phase concurrent flow in packed beds, Chem Eng Sci, 32: 515523 .

\section{ACKNOWLEDGEMENT}

The financial support from the National Science Council is gratefully acknowledged.

\section{ADDRESS}

Correspondence concerning this paper should be addressed to Professor Hwai-Shen Liu, Department of Chemical Engineering, National Taiwan University, Taipei, Taiwan, ROC, (E-mail: hsliu@ @ccms.ntu.edu.tw).

The manuscript was received 13 April 1999 and accepted for publication after revision 10 November 1999. 